\title{
Treatment Of Magnesium-L-Threonate Elevates The Magnesium Level In The Cerebrospinal Fluid And Attenuates Motor Deficits And Dopamine Neuron Loss In A Mouse Model Of Parkinson's disease
}

This article was published in the following Dove Press journal:

Neuropsychiatric Disease and Treatment

Yanling Shen, ${ }^{1,2, *}$ Ling Dai, ${ }^{1}, *$ Haibo Tian, ${ }^{1,3}$ Runnan Xu,' Fuying $\mathrm{Li}^{4}{ }^{4}$ Zhuohang $\mathrm{Li},{ }^{,}$ Jeremy Zhou, ${ }^{5}$ Liping Wang, (iD ${ }^{5}$ Jianghui Dong, (iD) ${ }^{1,5}$

Liyuan Sun ${ }^{1,4}$

'Department of Guangxi Key Laboratory of Brain and Cognitive Neuroscience, Guilin Medical University, Guilin 54I004, Guangxi, People's Republic of China; ${ }^{2}$ Department of Pathology, Affiliated Chenggong Hospital, Xiamen University, Xiamen, Fujian 361000, People's Republic of China; ${ }^{3}$ Department of Pathology, Fuling Central Hospital of Chongqing City, Chongqing 408099, People's Republic of China; ${ }^{4}$ Department of Neurology and Neurological Science, Tokyo Medical and Dental University, I-5-45 Yushima, Bunkyo-ku, Tokyo I I 3-85 I 9, Japan; ${ }^{5}$ School of Pharmacy and Medical Sciences, and UniSA Cancer Research Institute, University of South Australia, Adelaide, SA 500I, Australia

*These authors contributed equally to this work

Correspondence: Jianghui Dong Department of Guangxi Key Laboratory of Brain and Cognitive Neuroscience, Guilin Medical University, Guilin 541004, Guangxi, People's Republic of China

Tel +86-773-368065 I

Fax +86-773-3680230

Email Jianghui.dong@mymail.unisa.edu.au

Liyuan Sun

Department of Guangxi Key Laboratory of Brain and Cognitive Neuroscience, Guilin Medical University, Guilin 541004, Guangxi, People's Republic of China

Tel +86-773-5893516

Fax+86-773-5898939

Email sunly@glmc.edu.cn
Purpose: Epidemiology research has demonstrated that magnesium $(\mathrm{Mg})$ deficiency is associated with a high incidence of Parkinson's disease (PD). It is known that the systemic administration of $\mathrm{MgSO}_{4}$ is not able to elevate the $\mathrm{Mg}$ concentration in cerebrospinal fluid (CSF). This study aims to verify the protective effect of magnesium-L-threonate $(\mathrm{MgT})$ in 1methyl-4-phenyl-1, 2, 3, 6-tetrahydropyridine (MPTP) mouse model.

Methods: C57BL/6J mice were orally administered $\mathrm{MgT}$ or $\mathrm{MgSO}_{4}$ for 4 weeks, and received MPTP in the third week. After analysis of open-field and rotarod tests on the last day, tyrosine hydroxylase (TH) immunopositive cells and protein levels were quantified in the substantia nigra pars compacta $(\mathrm{SNpc})$ and striatum. The expression of inducible nitric oxide synthase (iNOS) level was evaluated. Mg concentration in serum and CSF was measured after oral administration of $\mathrm{MgSO}_{4}$ or $\mathrm{MgT}$ in normal mice. $\mathrm{Mg}$ concentration in the CSF was increased in the mice treated with $\mathrm{MgT}$ but not $\mathrm{MgSO}_{4}$.

Results: The total distance and mean speed in open-field tests, and the time spent on rotarod in the MgT group were increased, compared with MPTP group. The MgT treatment but not $\mathrm{MgSO}_{4}$ dose-dependently attenuated the loss of TH-positive neurons, and the reduction of the TH expression in the SNpc. The MgT treatment also inhibited the expression of iNOS as measured by immunohistochemistry and Western blots. Double-immunofluorescence staining of TH and iNOS showed iNOS-positive cells were collocalized for TH-positive cells.

Conclusion: The treatment with $\mathrm{MgT}$ is associated with an increase of $\mathrm{Mg}$ in the CSF. MgT, rather than $\mathrm{MgSO} 4$, can significantly attenuate MPTP-induced motor deficits and dopamine (DA) neuron loss.

Keywords: Parkinson's disease, magnesium-L-threonate, cerebrospinal fluid, magnesium

\section{Introduction}

Parkinson's disease (PD) is a neurodegenerative disease and its characterization includes muscular rigidity, bradykinesia, resting tremors, and postural instability, as well as several non-motor symptoms (Parkinson). ${ }^{1}$ Pathological features of PD are the progressive loss of dopamine producing neurons in substantia nigra (SN), cytoplasmic inclusions occur in surviving neurons of $\mathrm{SN}$, which are called Lewis bodies. ${ }^{1-3}$ The pathogenesis of PD may include a variety of factors, such as genetic factors or/and environmental factors. It is a relatively high incidence for agricultural workers when using herbicides and pesticides, particularly paraquat. 
One previously epidemiological study has demonstrated that the function of low-Mg diet in elective neurodegeneration of dopaminergic pathway is associated with Parkinson-dementia syndrome (PDC). ${ }^{4}$ The characterization of PDC involves progressive cognitive decline, parkinsonism and severe loss of neurons in the $\mathrm{SN}$ and widespread neurofibrillary tangles in the PDC brain. In addition, PDC is a deadly disease for the Chamorro people in Guam. High concentration of aluminum and low concentration of $\mathrm{Mg}$ and calcium in the water consumed by Chamorro natives have been reported for the high incidence of PD in Guam. ${ }^{5}$

To further investigate the pathogenesis of PDC, a study was designed to limit the intake of $\mathrm{Mg}$ and calcium in rats over two generations. The intention of the study was to simulate the conditions for humans on Guam. Severe loss of dopaminergic neurons in $\mathrm{SN}$ were found exclusively in 1-year-old rats that had taken a continuous intake of low $\mathrm{Mg}$ over generations. ${ }^{6}$ Another research evaluated the effect of MPTP in Mg-deficient mice, they found a low dose (like $10 \mathrm{mg} / \mathrm{kg}$ ) MPTP treatment can reduce the content of dopamine (DA) and its metabolites in striatum of Mg-deficient mice. It indicates Mg-deficiency appears to enhance sensitivity in MPTP neurotoxicity. ${ }^{7}$

Although the etiologic mechanism of PD related to Mg-deficiency is poorly understood, it can be assumed that hypermagnesemia can influence the development of experimental PD, because a low-Mg diet contributes to the high occurrence of PD. Hashimoto et al have proved that the toxicity of 1-methyl-4-phenylpyridinium $\left(\mathrm{MPP}^{+}\right)$could be significantly inhibited by increasing the concentration of $\mathrm{Mg}$ ions to $1.2 \mathrm{mM}$, and any reduction of dopaminergic neurons in in vitro MPP Parkinson's model can be completely prevented by increasing the concentration to $4 \mathrm{mM}^{8}$

Magnesium sulfate is a commonly used clinical medicine, and the first choice of clinical magnesium supplement (REF). Mainly intravenous magnesium sulfate has been used to investigate the neuroprotective effect of magnesium in preclinical and clinical studies. ${ }^{9-12}$ In a preclinical experiment, magnesium sulfate cannot play a neuroprotective role. ${ }^{13}$ In some clinical experiments, magnesium sulfate cannot improve the prognosis of patients with cerebral ischemia or subarachnoid hemorrhage. ${ }^{14-16}$ We speculate that the difference in the efficacy of magnesium sulfate is due to its poor permeability in the blood-brain barrier. Our previous study demonstrated that the increasing of $\mathrm{Mg}$ concentration in serum had no effect on the concentration of $\mathrm{Mg}$ in CSF after intraperitoneal injection of $\mathrm{MgSO}_{4}$, even when the serum $\mathrm{Mg}$ level increased from 8 to 10 fold in normal mice. ${ }^{17}$ Therefore, magnesium-L-threonate $(\mathrm{MgT}), \mathrm{ag}$ compound that is very permeable through the blood-brain barrier (BBB), ${ }^{18,19}$ was used in the present study. There was no adverse effect of $\mathrm{MgT}$ on normal rats. Biochemical analysis showed that the level of $\mathrm{Mg}$ in plasma increased significantly after $\mathrm{MgT}$ administration. Compared with the control group, the behavioral evaluation of elevated plus maze (EPM) test and forced swimming test (FST) showed that MgT could significantly improve the memory of rats and reduce the depression-like symptoms of healthy rats. ${ }^{20} \mathrm{We}$ measured $\mathrm{Mg}$ concentration in the CSF following chronic administration of $\mathrm{MgSO}_{4}$ or $\mathrm{MgT}$ in normal mice, evaluated behavioral analysis, DA neuron loss and expression of iNOS after oral $\mathrm{MgSO}_{4}$ or $\mathrm{MgT}$ in MPTP treatment mice.

The goal of this study was to demonstrate the neuroprotective effect of MgT on MPTP mice. It is well known that MPTP was the first agent to be distinguished for human $\mathrm{PD} .^{21}$ By means of monoamine oxidase $\mathrm{B}$ in astrocytes, MPTP can be converted to $\mathrm{MPP}^{+}$after systemic administration. $\mathrm{MPP}^{+}$interferes with the flow of electrons and damages mitochondrial complex I, which cause a severe shortage in ATP formation of dopaminergic neurons transferred from the dopamine transporter.

\section{Materials And Methods}

For the care and use of animals in research, this study was implemented according to NIH guidelines. ${ }^{22}$ The procedures of animal experiment in this study were approved by the Animal Care and Use Committee of Guilin Medical University.

\section{Animals}

Male C57BL/6J mice ( $\mathrm{n}=87$, Vital River Laboratory Animal Technology Co. Ltd, Beijing, People's Republic of China), 8 weeks old with 20 to $22 \mathrm{~g}$, were used. All mice were fed separately, and they were freely provided with water and food in the light and dark cycle of 12:12 hrs, and light was given at 8:00 pm.

\section{Drugs}

MPTP was purchased from Sigma (St. Louis, MO, USA) and then diluted with saline $(0.9 \% \mathrm{NaCl})$ at a concentration of $4 \mathrm{mg} / \mathrm{mL}$. Two $\mathrm{Mg}$ compounds: $\mathrm{MgSO}_{4} \cdot 7 \mathrm{H}_{2} \mathrm{O}$ (Rong Sheng Pharmaceutical Co., Ltd, People's Republic 
of China) and MgT (Magceutics Inc., USA) were used in the current study.

\section{Experimental Protocols}

In order to examine the effect of $\mathrm{Mg}$ compounds in the mice model, the behavioral difference and $\mathrm{Mg}$ concentration in CSF and serum were measured after the individual treatment of $\mathrm{MgSO}_{4}$ or $\mathrm{MgT}$. Normal mice received 1.2 $\mathrm{mM} \mathrm{MgSO}_{4}$ or $\mathrm{MgT}$ via drinking water throughout the experiment. Behavioral tests and $\mathrm{Mg}$ assays were conducted on day 0,21 , and 28 ( $n=6$ in each subgroup).

To detect neuroprotective effects of $\mathrm{MgSO}_{4}$ or $\mathrm{MgT}$, the mice were separated into four groups randomly: 1) control group, fed the basal diet, $\mathrm{n}=12$; 2) MPTP group, mice were intraperitoneally injected $30 \mathrm{mg} / \mathrm{kg} /$ day MPTP$\mathrm{HCl}$ with saline for successive 7 days after 2 weeks of the experiment start, $\mathrm{n}=12$; 3) $\mathrm{MgSO}_{4}$-MPTP group, mice received $\mathrm{MgSO}_{4}(1.2 \mathrm{mM})$ via drinking water throughout the experiment, and were injected with MPTP-HCl, as the same as the MPTP group, $n=12$; 4) MgT-MPTP groups, mice received 3 different doses of $\operatorname{MgT}(0.8,1.2$, and 1.6 $\mathrm{mM}$ ) by oral administration in three subgroups, and were injected with MPTP-HCl as the same as the MPTP group, $\mathrm{n}=12$, in each subgroup. The dose of two $\mathrm{Mg}$ compounds was chosen by adjusting the minimum effective dose in mice, the description can be found in the previous study. ${ }^{19}$

To observe the dose of $\mathrm{MgSO}_{4}$ and $\mathrm{MgT}(\mathrm{mm} / \mathrm{kg} /$ day), it is necessary to measure body weight and water intake daily $(8: 30 \mathrm{pm})$. Based on the amount of water consumption daily and body weight, the amount of $\mathrm{MgSO}_{4}$ and MgT was obtained to ensure the target doses, and then the daily drinking water for each mouse (about $6 \mathrm{~mL} / \mathrm{mouse} /$ day) was used to dissolve the drug.

\section{Measurement Of $\mathrm{Mg}$ Concentration}

In order to collect the serum of the mice model, 1 to $1.5 \mathrm{~cm}$ was dissected from the tip of the tail, and polypropylene tips (catalog no. RS-200Y, Renover Science, Co., Ltd., Tokyo, Japan) was used to collect blood with a polypropylene tube (catalog No. 430791, Corning, NY, USA). CSF was achieved through the cisterna magna. The tubes were placed into the centrifuge at $1500 \mathrm{rpm}$. The muscle and skin in the neck were separated, and then the exposed arachnoid membrane and dura mater were penetrated with a butterfly needle $(27 \mathrm{G} \times 1 / 2,0.4 \times 13 \mathrm{~mm}$, TOP Co., Tokyo, Japan) to inhale CSF. The Milli-Q water was used to dilute the samples at 1000 -fold.
Quadrupole ICP-MS (7500 Series, Agilent Technologies, Tokyo, Japan) was inductively coupled plasma mass spectrometry and used to measure the concentration of $\mathrm{Mg}$. For internal standardization, the samples were mixed in $1 \%$ nitric acid with Yttrium (Kanto Kagaku, Co., Ltd., Tokyo, Japan), and the final concentration was $10 \mu \mathrm{gL}^{-1}$. Clean the instrument with Milli-Q water before adding the sample or normalizing. The working conditions of ICP-MS were given: argon flow rate, carrier $0.35 \mathrm{~L} / \mathrm{min}$, plasma $0.7 \mathrm{~L} / \mathrm{min}$; cooling chamber temperature at $2^{\circ} \mathrm{C}$; ICP RF power with $1500 \mathrm{~W}$; scanning quality, $\mathrm{y} \mathrm{m} / \mathrm{z}=89 \mathrm{mg}, \mathrm{m} / \mathrm{z}=25$; hydrogen flow rate, 0.4 . Each standard solutions or sample solutions were measured with 3 times.

\section{Behavioral Analysis}

Open-field and rotarod tests were used to measure the locomotor activity and motor coordination dysfunction of mice for 7 days after the last MPTP injection. Before any treatment, mice were exposed to the adaptive training with 2-3 times.

The automatic open-field apparatus was performed to evaluate open-field test-spontaneous locomotor activity. ${ }^{23,24}$ An open-field box $(60 \times 60 \times 50 \mathrm{~cm})$ divided into 16 squares was conducted to assess the spontaneous activity of mice. Mice were placed in the open-field apparatus and kept for 5 mins subjected to normal lighting. ANY-maze software (Shanghai new soft information technology Co., Ltd, People's Republic of China) was used to investigate the trajectories and movements of mice based on video-taped. To remove the body scent, water and 70\% alcohol were used to clean the box after each testing. Mice were put in the open-field box for $1 \mathrm{~min}$ to adapt to the venue before any treatments.

Rotarod test-Refer to test Rozas method, ${ }^{24,25}$ mice were placed on a rotarod $3 \mathrm{~cm}$ in diameter, which rotated along its longitudinal axis at four speeds $(10,12,14,16$ rpm). The time spent on the rotarod before falling was recorded. The trial stopped when the mouse fell down or when $150 \mathrm{~s}$ were completed, and there was an interval of 5 mins between trials to reduce stress and fatigue. The average time on the rotarod from the five trials was used for analysis. The effects of drug treatments were assessed by testing the various groups of animals at the same time after injection.

\section{Histological Preparation}

Paraffin tissue and frozen tissue were prepared in two time points: the next day after the last MPTP (day 21) treatment 
to evaluate the expression of iNOS; the last day (day 28) after end of the behavioral experiments to assess the expression of TH. For paraffin tissue, Nembutal $(50 \mathrm{mg} /$ kg, Dainippon Pharmaceutical Co., Ltd.) was used to anesthetize mice, and then $4 \%$ phosphate buffer polyformaldehyde (PFA) solution was used to perfuse and fix through heart. The brain of mouse was coronally cut at $3 \mathrm{~mm}$ posterior to the bregma (superior colliculus level), $1.5 \mathrm{~mm}$ posterior to the bregma (infundibular level) and $0.5 \mathrm{~mm}$ anterior to the bregma (chiasmal level). The obtained blocks were embedded in paraffin and cut with 4 microns thickness. The resulting blocks were put in paraffin and cut into slice with $4 \mu \mathrm{m}$. For frozen tissue, mice were perfused with $4 \%$ PFA, the brains of mice were processed into blocks as mentioned above with dehydration using different concentrations of sucrose (low to high); they were then mounted in OCTA mold filling and sliced with $16 \mu \mathrm{m}$ thickness.

\section{Immunohistochemistry}

The sections were immersed in $\mathrm{H}_{2} \mathrm{O}_{2}(3 \%)$ for 30 mins, and washed with PBS. The normal goat serum (10\%) was used to treat the slices for $30 \mathrm{mins}$, and then the slices were incubated by a mouse IgG secondary antibody or biotinylated goat anti-rabbit for 60 mins. To observe the binding antibody, the biotin-biotinylated peroxidase complex (ABC) method was used. After 60 mins, the peroxidase reaction was carried out in diaminobenzidine (DAB). The numbers of immunopositive in SN were determined by a microscope attached with a drawing tube (Nikon, Tokyo, Japan) at $200 \times$ magnification. In this study, the experimental conditions of each histological specimen were blinded to the researchers.

The primary antibodies, including rabbit anti-iNOS (Chemicon, Temecula, CA, USA; 1:2000 dilution) and mouse anti-tyrosine hydroxylase (Chemicon, Temecula, CA, USA; 1:2000 dilution) were used in this study.

For double-immunofluorescence staining of TH and iNOS, the slices were put in PBS with $10 \%$ NGS at room temperature for 60 mins and incubated with mouse anti-tyrosine hydroxylase and rabbit anti-iNOS overnight at $4{ }^{\circ} \mathrm{C}$. Subsequently, slices of brains were washed by PBS, and incubated in Alexa 594-coupled anti-mouse IgG and Alexa 488-coupled anti-rabbit IgG for $2 \mathrm{hrs}$ at room temperature. Double positive cells were examined with a Zeiss M510 laser scanning confocal imaging system.

\section{Western Blotting Analysis}

To assess the protein expression of iNOS (day 21) and TH (day 28), control-, MPTP-, and $1.2 \mathrm{mM} \mathrm{MgT}$ mice were prepared $(n=6)$. The dissected tissues were well distributed in ice-cold Tris- $\mathrm{HCl}$ buffer with $25 \mathrm{mM}$, which included protease inhibitors (benzenesulfonyl fluoride, bestatin, leupeptin, and aprotinin) and $2 \mathrm{mM}$ EDTA. The homogenate was centrifuged with $11 \mathrm{~kg}$ for 5 mins at $4^{\circ} \mathrm{C}$, the pellet was resuspended in fresh buffer (sodium dodecyl sulfate, Tris, h-mercaptoethanol, and glycerol), and decentered with $20 \mathrm{~kg}$ for $30 \mathrm{mins}$. The source of proteins was obtained from the supernatant, and the concentration of protein was evaluated by Coomassie blue protein assay. The protein was electrophoresed on polyacrylamide gels, shifted to the membrane of nitrocellulose, and detected by polyclonal affinity purified anti-iNOS, anti-Th antibodies, and HRP coupled IgG, respectively. Subsequently, ECL Western blotting kit (Amersham Pharmacia Biotech, Piscataway, NJ) was used to observe the immunoreactive protein on the filter NIH image program was used to quantify the Western blot intensity by density scanning, and the recombinant beta-actin signal was normalized on the same Western blot.

\section{Statistical Analysis}

The data were examined using repeated measurement variance analysis (ANOVA), and the testing day was taken as an independent variable. Bonferroni postmortem multiple comparison test was used to analyze the groups. All results are illustrated with mean \pm SD. Statistical significance level was set at $\mathrm{P}<0.05$.

\section{Results}

\section{Treatment Of $\mathrm{MgSO}_{4}$ Or MgT In Normal Mice}

There were no significant changes in behavioral analysis when individual treatment of $\mathrm{MgSO}_{4}$ or $\mathrm{MgT}$ in normal mice. $\mathrm{Mg}$ concentrations in serum and CSF were $1.38 \pm 0.12$ $\mathrm{mM}$ and $0.89 \pm 0.11 \mathrm{mM}$, respectively. It was found that there was no obvious change in the CSF that was treated with $\mathrm{MgSO}_{4}$, the concentration of $\mathrm{Mg}$ in the serum was remarkably promoted to $2.24 \pm 0.18 \mathrm{mM}$ from $1.38 \pm 0.12$ $\mathrm{mM}$ on day 21 , and reached $2.21 \pm 0.19 \mathrm{mM}$ on day 28 . However, the concentration of $\mathrm{Mg}$ in the CSF increased significantly from $0.89 \pm 0.11 \mathrm{mM}$ to $1.15 \pm 0.15 \mathrm{mM}$ on day 21 , and $1.17 \pm 0.16 \mathrm{mM}$ on day 28 after the treatment with $1.2 \mathrm{mM} \mathrm{MgT}$ (Figure 1). 


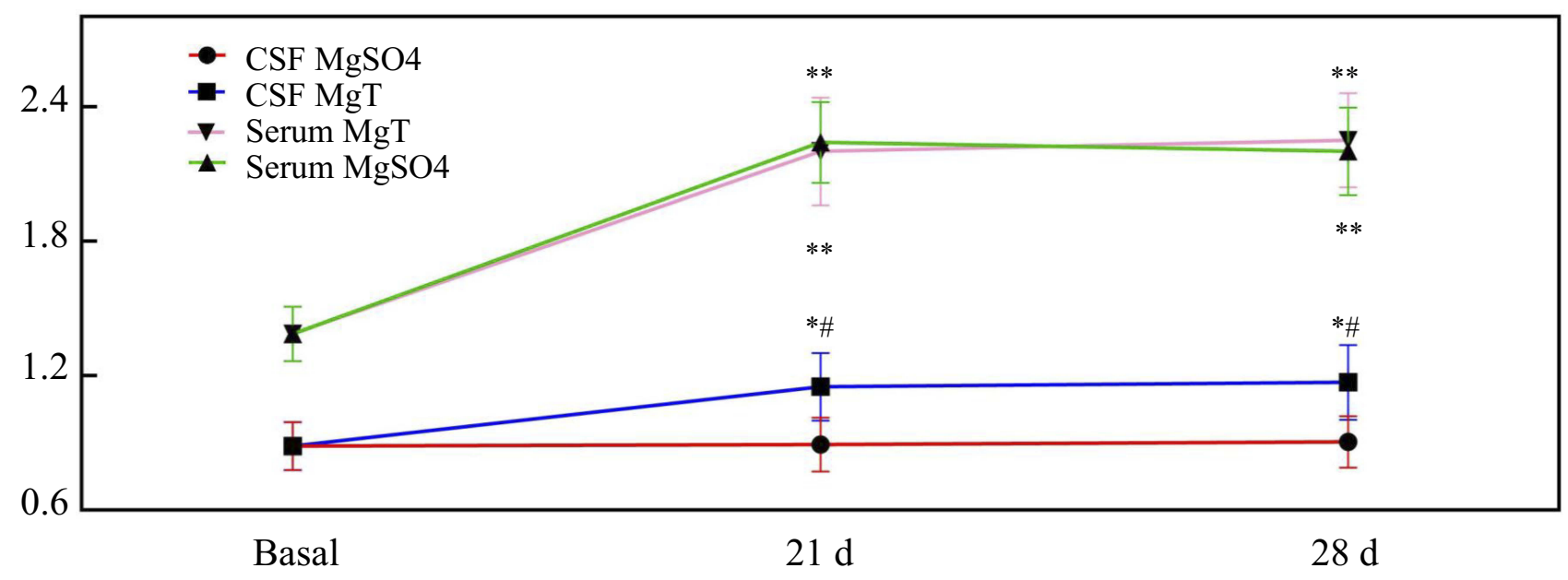

Figure I The changes of magnesium concentration in cerebrospinal fluid (CSF) and serum following treatment with $\mathrm{MgSO}_{4}$ or $\mathrm{MgT}$ on day 0, $2 \mathrm{I}$, and 28 in normal mice. $* P<0.05, * * P<0.01$, compared with basal, ${ }^{\#} P<0.05$, compared with $\mathrm{MgSO}_{4}$ group.

\section{MgT Improves Motor Coordination And Motor Dysfunction In MPTP-Injected Mice}

Representative motor activity maps of movement of each treatment group mice (day 28) are shown in Figure 2A. Compared with the control group mice, the total distance and mean speed in MPTP group mice decreased 37.6\% and $39.7 \%$, respectively (Figure $2 \mathrm{~B}$ and $\mathrm{C}$ ). However, compared with MPTP group, the total distance and mean speed in $1.2 \mathrm{mM} \mathrm{MgT}$ treated group were significantly increased $38.5 \%$ and $50.1 \%$, respectively, respectively. The distance and time that mice travelled in the center decreased from $57.1 \%$ and $46.5 \%$ in MPTP group mice, however, there were no difference between all treatment groups and MPTP group (Figure 2C).

In the rotarod test, the rotarod time of MPTP group was significantly shorter in 12,14 , and $16 \mathrm{rpm} / \mathrm{min}$ than that of the control group but no change was found in 10 $\mathrm{rpm} / \mathrm{min}$ test. The MgT treatment dose-dependently increased the rotarod time in $14 \mathrm{rpm} / \mathrm{min}$ test.

\section{DA Neurons In SN}

The value of TH-positive neurons (Figure 3A, inset) of SNpc was remarkably decreased for MPTP group (62.4\% of the control group). As shown in Figure $3 \mathrm{~A}$ and $\mathrm{B}$, the $\mathrm{MgT}$ treatment dose-dependently attenuated the loss of $\mathrm{TH}^{+}$neurons compared to the MPTP group. TH-positive neurons were observed also in ventral tegmental area (VTA) in normal mice, and well preserved in MPTP group, and no significant change was found in MgT or $\mathrm{MgSO}_{4}$ treatment group. The analysis of Western blot demonstrated that the protein bands of $\mathrm{TH}$ deduced at $31.3 \%$ in $\mathrm{SN}$ and $76.1 \%$ in striatum (Figure $3 \mathrm{C}$ and $\mathrm{D}$ ) in MPTP treated mice, and the MgT treatment (1.2 mM) attenuated the reduction of $\mathrm{TH}$, reaching $18.9 \%$ and $65.9 \%$ in $\mathrm{SN}$ and striatum of the control, respectively.

\section{Expression Of iNOS In The SN}

The expression of iNOS level was evaluated the next day (day 21) after MPTP treatment for 7 days. Compared with the control group, the number of iNOS-positive cells of the MPTP group was remarkably increased, and the number of iNOS-positive cells in the $1.2 \mathrm{mM} \mathrm{MgT}$ treatment significantly decreased (Figure 4). The analysis of Western blot illustrated that the MPTP treatment enhanced the expression of iNOS in SN by $55.6 \%$ (Figure 4B) and the MgT treatment at $1.2 \mathrm{mM}$ significantly decreased iNOS expression. Double-immunofluorescence staining of $\mathrm{TH}$ and iNOS showed most of the iNOS-positive cells were THpositive DA neurons (Figure 5).

\section{Discussion}

$\mathrm{Mg}$ has a basic role in the function of cell and its deficiency is associated with neurological disorders. The administration of intravenous $\mathrm{MgSO}_{4}$ has a neuroprotective influence on cerebral ischemia, subarachnoid hemorrhage, seizure and traumatic brain injury in both animals and humans based on clinical and experimental studies. ${ }^{24,26}$ Although many reports have investigated that serum $\mathrm{Mg}$ can enter $\mathrm{CSF}$, the ionic composition in the CSF keeps significantly stable when blood ionic composition with variation in animals ${ }^{27-29}$ or humans ${ }^{30}$ subjected to the normal pathologic conditions. The 
A

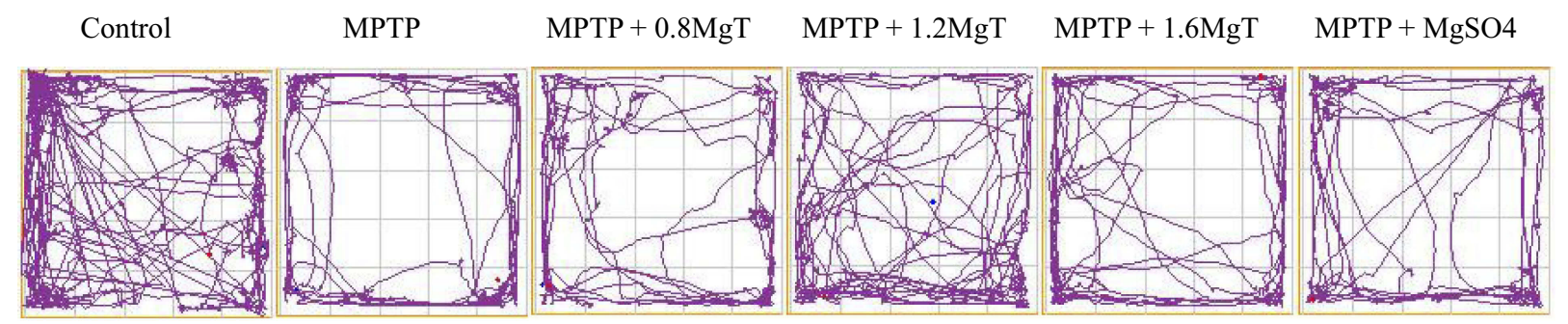

B

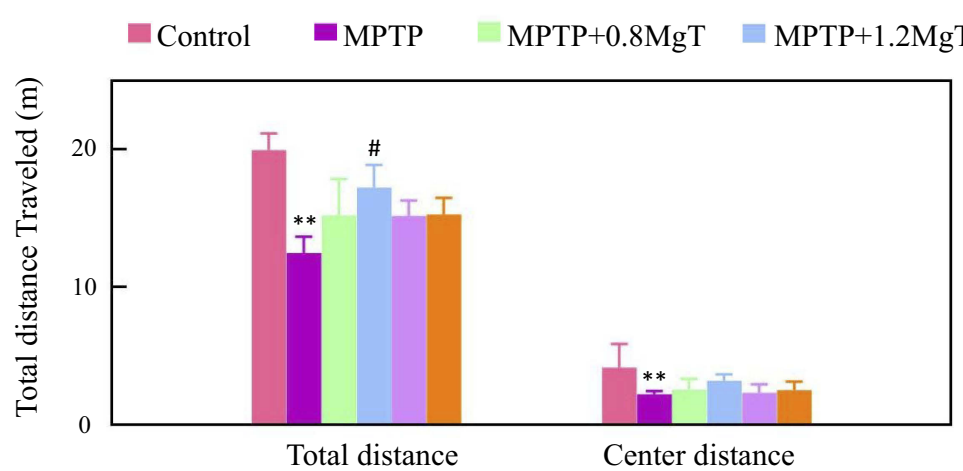

D
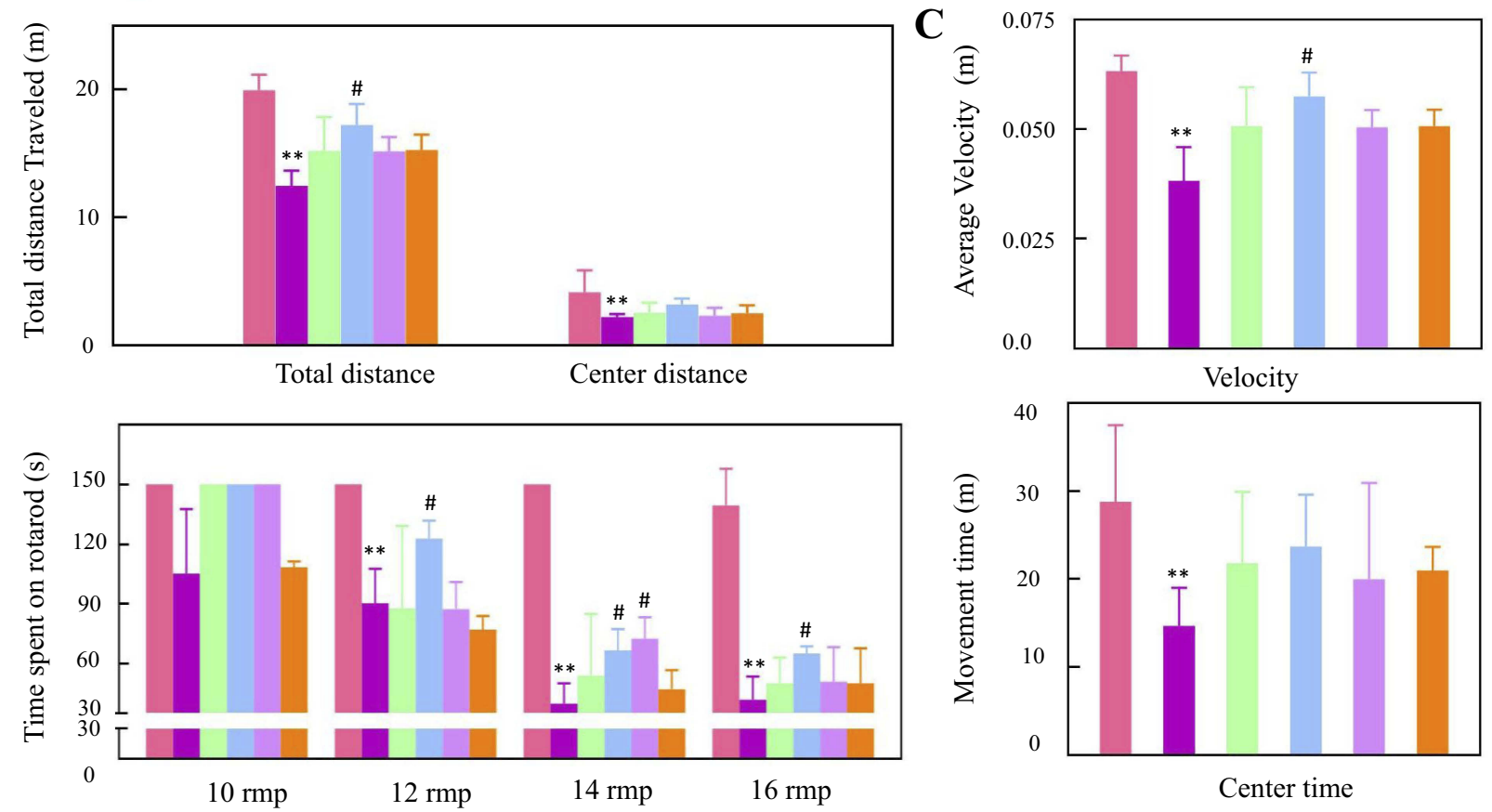

Figure 2 Open-field and rotarod tests. (A) Representative motor activity maps of mouse movement from each treatment group (day 28); (B) total distance; (C) average velocity and movement time; (D) rotarod time in 10, 12, 14, and $16 \mathrm{rpm} / \mathrm{min}$, respectively. $* * P<0.01$, compared with control. \#P<0.05, compared with MPTP group.

serum $\mathrm{Mg}$ level increased 2-4 times in dogs under the normal conditions, while $\mathrm{Mg}$ level in the CSF enhanced slightly in patients with brain injury. ${ }^{31}$ However, $\mathrm{Mg}$ level in CSF in preeclampsia patients and hippocampal epilepsy rats increased remarkably, indicating the CSF Mg level is actively regulated, possible by $\mathrm{Mg}$ transporter located in $\mathrm{BBB} .{ }^{32}$ Our previous study also demonstrated that concentration of $\mathrm{Mg}$ in the CSF had no any variation with the raise of the $\mathrm{Mg}$ concentration in serum, even when the serum $\mathrm{Mg}$ level increased 8- to 10 -fold in normal mice. ${ }^{17}$ Here, our results showed that $\mathrm{MgT}$, but not $\mathrm{MgSO}_{4}$, increased the CSF $\mathrm{Mg}$ level. We also showed that $\mathrm{MgT}$, but not $\mathrm{MgSO}_{4}$ can significantly attenuate MPTP-induced motor deficits and DA neuronal degeneration in mice. These results indicated that the positive influence of MgT can be associated with the elevation of $\mathrm{Mg}$ concentration in the CSF after treatment with $\mathrm{MgT}^{33}$ The Mg concentration increased about $21.6 \%$ after treatment of MgT on day 28 in CSF, which were concordant with the previous data that $\mathrm{Mg}$ concentration was increased to $15 \%$ in rat CSF and increased to $30 \%$ in APPswe/PS1dE9 mice brain tissue after oral MgT. ${ }^{19,34}$

$\mathrm{Mg}$-deficiency may be related with the pathogenesis of PDC that occurs in the Chamorro people of Guam, and rats with $\mathrm{Mg}$ deficiency for generations with severe loss of DA neurons, ${ }^{35}$ and the sensitivity of mice to MPTP neurotoxicity seems to enhance. ${ }^{7}$ However, the mechanism of $\mathrm{PD}$ related to $\mathrm{Mg}$-deficiency is poorly understood. The deficiency of $\mathrm{Mg}$ is related to decreased antioxidant defense and increased oxidative stress in different tissues based on animal and human models. ${ }^{18,31,36}$ In animals, Mg-deficiency resulted in a marked promotion of some pro-inflammatory molecules, such as VCAM, I IL-6, L-1b, PAI-1, and TNF-a. ${ }^{37,38}$ Inflammatory cytokines can induce free radical generation and promote neuronal degeneration by causing oxidative stress. In $\mathrm{SN}$, the microglia is densest and the number is $4-5$ times higher than in other regions in 


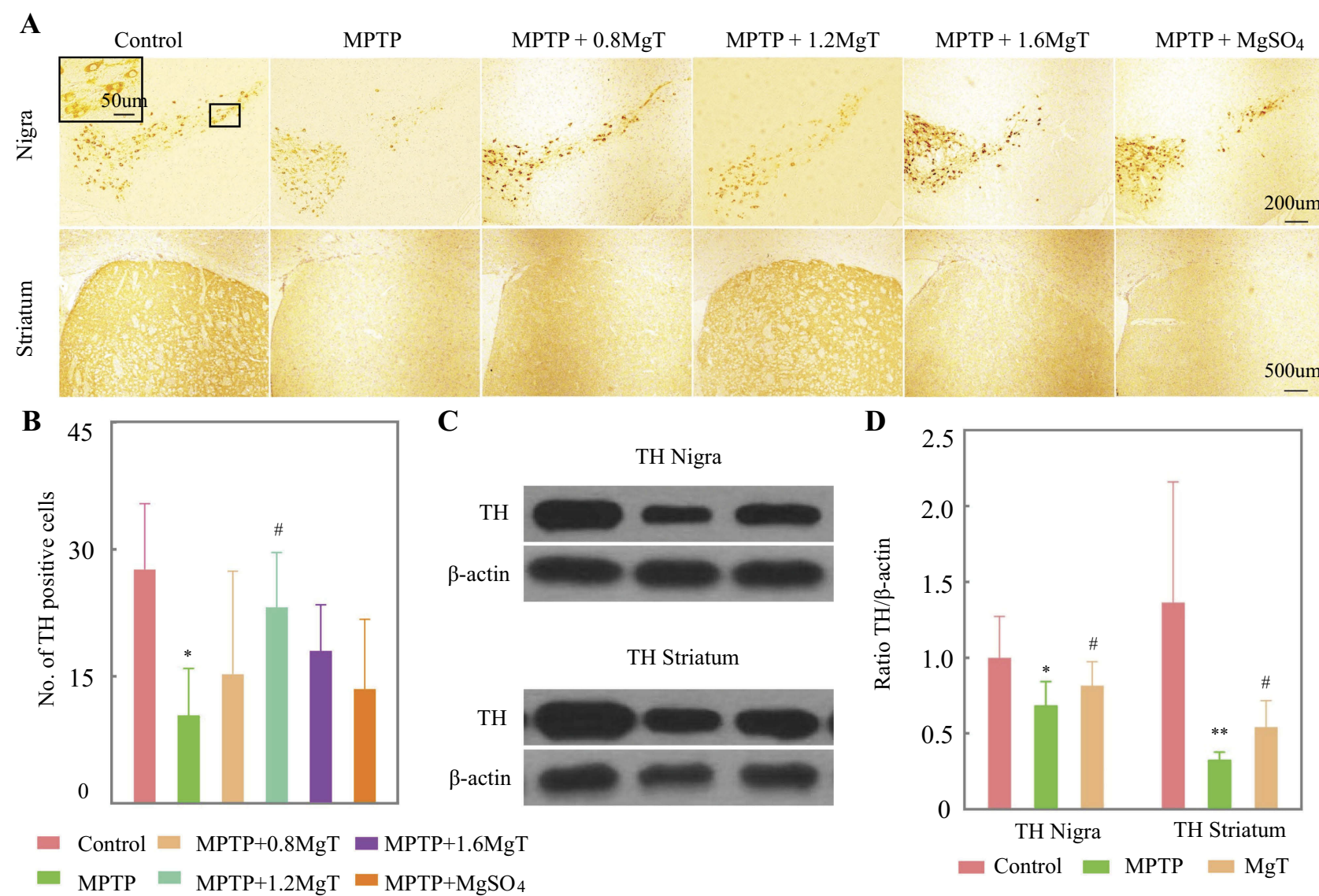

Figure 3 Immunohistochemical staining and Western blot analysis of TH. (A) Representative light micrographs of immuno-staining of TH in SN and striatum; (B) number of TH-positive cells in SNpc; (C) Western blot analysis of TH in SN and striatum. Upper row showed representative gel bands for TH protein, lower row showed $\beta$-actin signals from the same blot; (D) ration $\mathrm{TH} / \boldsymbol{\beta}$-actin. ${ }^{*} \mathrm{P}<0.05$, $* * \mathrm{P}<0.01$, compared with control, \#P<0.05, compared with MPTP group.

the central nervous system, therefore, it is conceivable that DA neurons in the $\mathrm{SN}$ are selective vulnerable when exposed continuously to low $\mathrm{Mg}$ intake.

MPTP was a neurotoxin and recognized early in 1982 as the first human parkinsonian agent was identified. MPTP generates a severe and irreversible Parkinson's syndrome, which almost duplicates the characteristics of PD. ${ }^{39}$ After systemic administration of MPTP, it is converted to $\mathrm{MPP}^{+}$ in astrocytes, released into extracellular space and freely entered into DA neurons by dopamine transporter. $\mathrm{MPP}^{+}$ damages mitochondrial complex I, interrupts the flow of electrons, and causes a severe shortage in the formation of ATP. The other result of mitochondrial complex I blocked from $\mathrm{MPP}^{+}$is an enhanced result of reactive oxygen species (ROS), which produce toxic effects with other active substances like nitric oxide synthase (NO) generated from inducible NO synthase (iNOS) and neuronal NO synthase (nNOS) in the brain. ${ }^{39,40}$

The neurotoxic of NO may affect its function in excitotoxity, neuroinflammation, DNA damage, oxidative stress, and mitochondrial dysfunction. Mice knocked out of $\mathrm{nNOS}^{41}$ or iNOS gene illustrated a stronger resistance to MPTP than wild-type mice. Administration of MPTP to mice induces upregulation of iNOS, and increases immunoreactivity for nNOS in the SN. ${ }^{42}$ Pretreatment inhibitor of microglial activation inhibits nigrostriatal dopaminergic neuronal degeneration and formation of nitrotyrosine evoked from MPTP. ${ }^{43-46}$ In this study, we demonstrated the treatment of MgT attenuated the loss of TH-positive neurons and the reduction of $\mathrm{TH}$ protein in SNpc. The mechanism underlying the $\mathrm{Mg}$ effect remains unclear. One previous study suggested that $\mathrm{Mg}$ in enough content inhibits the formation of oxygen radical from scavenging free radicals and preventing oxidase of both NADPH and xanthine. ${ }^{47}$ Moreover, $\mathrm{Mg}$ is required for normal functioning of the nervous system. It is a cofactor in hundreds of enzymatic reactions, such as activation of phosphotransferase and hydrolases (like ATPase), which are of central importance in the biochemistry of the cell, particularly energy metabolism. $\mathrm{Mg}$ concentrates ribosomes and is 

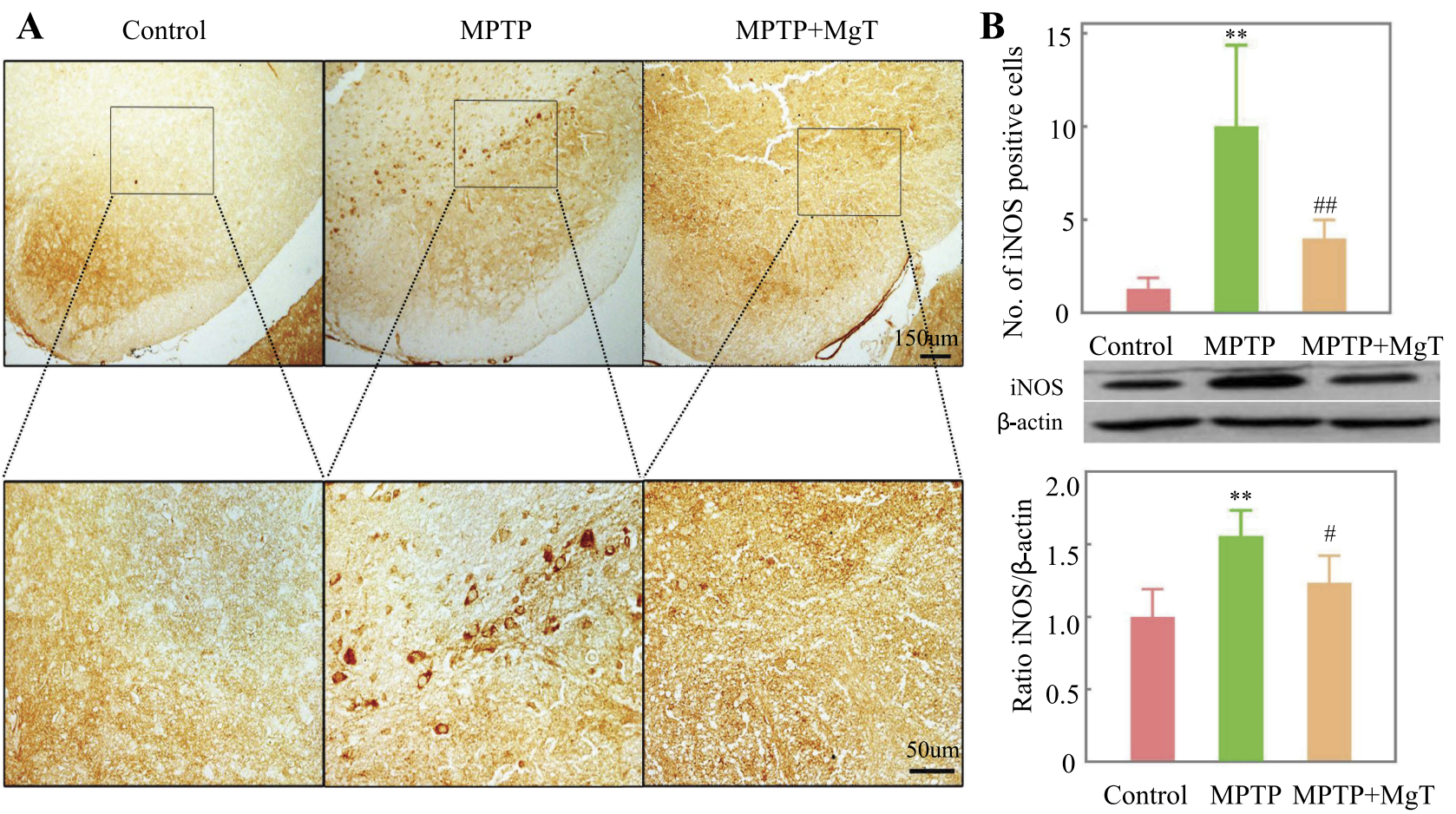

Figure 4 Immunohistochemical staining (A) and Western blot analysis of iNOS in SN; (B) representative light micrographs of immuno-staining of iNOS in SN were given in (A) quantitative analysis of iNOS-positive cells was presented in (B) $* * P<0.01$, compared with control; ${ }^{\#} P<0.05$, ${ }^{\# P} P<0.01$, compared with MPTP group.

involved in the attachment of mRNA to them. Thus, Mg is required for protein and nucleic acid synthesis, cell cycle activity, cytoskeletal and mitochondrial integrity, and for binding of substances to the plasma membrane. In addition, iNOS activated by proinflammatory cytokines and Gram-negative endotoxins was increased in glial cells in MPTP model. ${ }^{37,48,49}$ Our study also observed active microglia and astrocytes in $\mathrm{SN}$, and the iNOS upregulation and co-localization with TH-positive cells in SNpc by laser scanning microscopy (Figure 5), which were inhibited from MgT.

Our results also showed the administration of $\mathrm{MgSO}_{4}$ had a marginal effect on mean speed induced in open-field test, but had no effect on neurodegeneration. Center time and center distance were generally regarded as an indicator of anxiety. A previous study has evaluated the effect of chronic administration of $\mathrm{MgSO}_{4}$ in MPTP mice, and shows that the administration of $\mathrm{MgSO}_{4}$ with low dose (like $2.5 \mathrm{~g} / \mathrm{l}$ ) generated a raise in latency and motor activity under thermal stimuli. ${ }^{50}$ However, the exhaustion of dopamine in striatum induced by MPTP treatment was not improved, and even exacerbated by high doses of $\mathrm{MgSO}_{4}$. A number of factors are involved in anxiety disorders, such as serotonin, dopamine, and other neurotransmitter system perturbations, ${ }^{51}$ and more than $40 \%$ of patients with PDcould have the clinical anxiety caused by the above factors. ${ }^{52}$ There was no difference in light-dark preference test in mice with 7 days after MPTP injury, ${ }^{53}$ and DA depletion was imperative but not enough to influence anxiety disorder. ${ }^{23}$

In the current study, the data suggest that the beneficial effect of $\mathrm{MgT}$ on neuroprotection is likely due to its effect on the Mg permeation into the brain, as the administration of $\mathrm{MgT}$ increased the concentration of $\mathrm{Mg}$ in both the CSF and serum. On the other hand, $\mathrm{MgSO}_{4}$ had no effect on the neurodegeneration as it only increased the serum Mg level but did not affect the CSF Mg level. These data also suggest that the only supplementation of $\mathrm{Mg}$ in the periphery does not help to protect the brain and the combination of $\mathrm{Mg}$ with an agent that promotes its transportation to the brain is essential for the neuroprotection of this element.

\section{Conclusion}

In conclusion, our results indicate $\mathrm{MgT}$ can significantly attenuate MPTP-induced motor deficits and DA neuron injury, which may be related to its ability of increasing the $\mathrm{Mg}$ concentration in the CSF. 

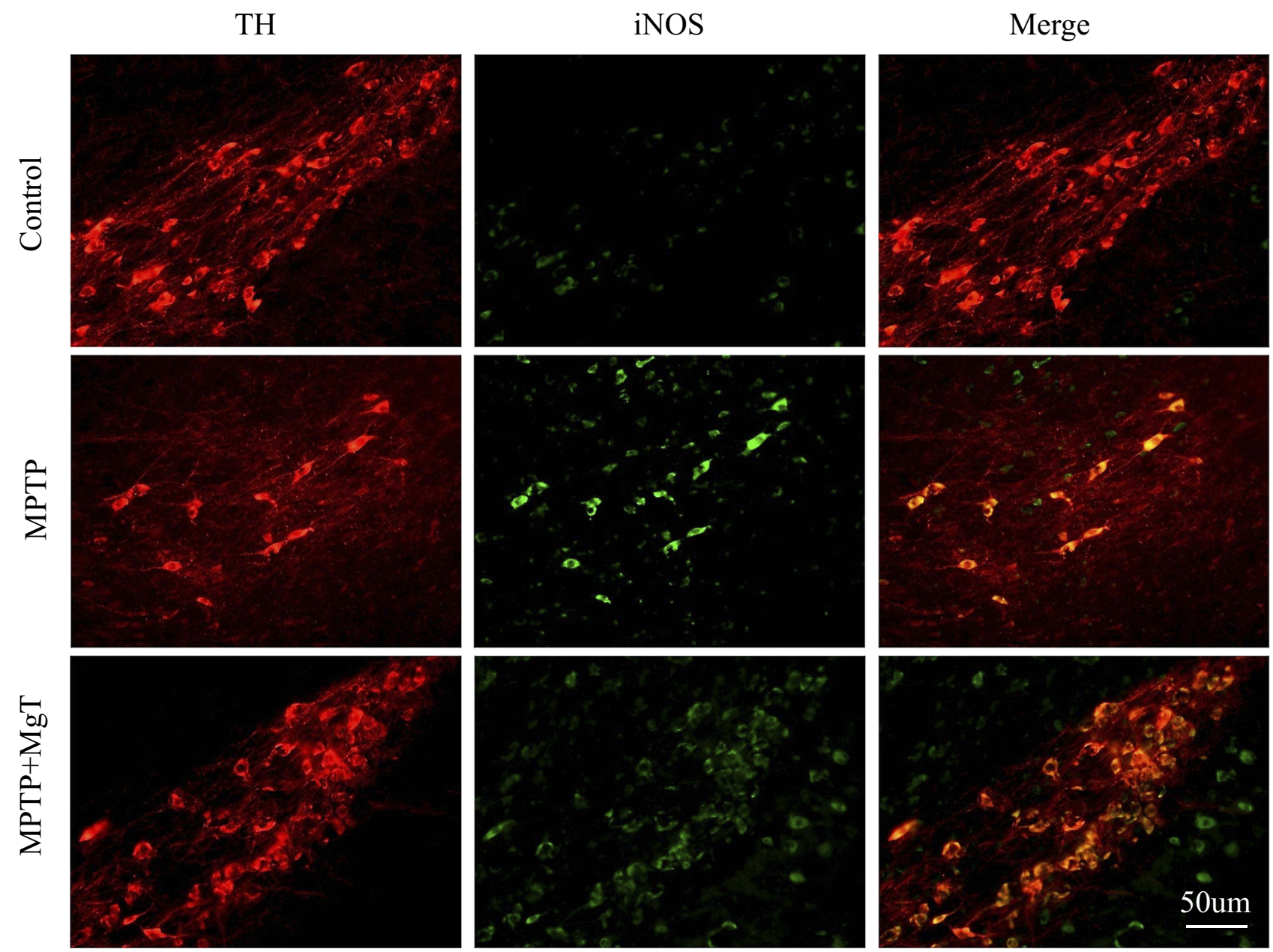

Figure 5 The results of immunofluorescence staining of iNOS/TH. Representative micrographs indicate most of the iNOS-positive cells were TH-positive DA neurons.

\section{Acknowledgments}

This work was supported by the National Natural Science Foundation of China (81260205) and Guangxi Natural Science Foundation (2018GXNSFAA281246).

\section{Disclosure}

The authors report no conflicts of interest in this work.

\section{References}

1. De Virgilio A, Greco A, Fabbrini G, et al. Parkinson's disease: autoimmunity and neuroinflammation. Autoimmun Rev. 2016;15(10):10051011. doi:10.1016/j.autrev.2016.07.022

2. González-Casacuberta I, Juárez-Flores DL, Morén C, Garrabou G. Bioenergetics and autophagic imbalance in patients-derived cell models of parkinson disease supports systemic dysfunction in neurodegeneration. Front Neurosci. 2019;13:894. doi:10.3389/fnins.2019.00894

3. Vila M, Przedborski S. Genetic clues to the pathogenesis of parkinson's disease. Nat Med. 2004;10(Suppl):S58-S62. doi:10.1038/nm1068

4. Taniguchi R, Tan-no K. Combined low calcium and lack magnesium is a risk factor for motor deficit in mice. Biosci Biotechnol Biochem. 2013;77(2):4. doi:10.1271/bbb.120671
5. Li Y, Jiao Q, Xu H. Biometal dyshomeostasis and toxic metal accumulations in the development of alzheimer's disease. Front Mol Neurosci. 2017. doi:10.3389/fnmol.2017.00339

6. Oyanagi K, Hashimoto T. Magnesium in Parkinson's Disease: An Update in Clinical and Basic Aspects. South Australia: University of Adelaid Press; 2011.

7. Muroyama A, Inaka M, Matsushima $\mathrm{H}$, Sugino $\mathrm{H}$, Marunaka $\mathrm{Y}$, Mitsumoto Y. Enhanced susceptibility to MPTP neurotoxicity in magnesium-deficient C57BL/6N mice. Neurosci Res. 2009;63 (1):72-75. doi:10.1016/j.neures.2008.09.009

8. Hashimoto T, Nishi K, Nagasao J, et al. Magnesium exerts both preventive and ameliorating effects in an in vitro rat parkinson disease model involving 1-methyl-4-phenylpyridinium (MPP+) toxicity in dopaminergic neurons. Brain Res. 2008;1197(p):9. doi:10.1016/j.brainres.2007.12.033

9. Shindo Y, Yamanaka R, Suzuki K, Hotta K, Oka K. Intracellular magnesium level determines cell viability in the MPP+ model of parkinson's disease. Biochim Biophys Acta Mol Cell Res. 2015;1853(12):3182-3191. doi:10.1016/j.bbamcr.2015.08.013

10. Bennet L, Galinsky R, Draghi V, et al. Time and sex dependent effects of magnesium sulphate on post-asphyxial seizures in preterm fetal sheep. $J$ Physiol. 2018;596(23):6079-6092. doi:10.1113/tjp.2018.596.issue-23

11. Saver JL, Starkman S, Eckstein M, et al. Methodology of the Field Administration of Stroke Therapy-Magnesium (FAST-MAG) phase 3 trial: part 2-prehospital study methods. Int J Stroke. 2014;9(2):220225. doi:10.1111/ijs. 12242 
12. Huang Y, Huang X, Zhang L, et al. Magnesium boosts the memory restorative effect of environmental enrichment in alzheimer's disease mice. CNS Neurosci Ther. 2018;24(1):70-79. doi:10.1111/cns.2018.24. issue-1

13. Zhu H-D, Martin R, Meloni B, et al. Magnesium sulfate fails to reduce infarct volume following transient focal cerebral ischemia in rats. Neurosci Res. 2004;49(3):347-353. doi:10.1016/j.neures.2004.04.001

14. Reddy D, Fallah A, Petropoulos J-A, Farrokhyar F, Macdonald RL, Jichici D. Prophylactic magnesium sulfate for aneurysmal subarachnoid hemorrhage: a systematic review and meta-analysis. Neurocrit Care. 2014;21(2):356-364. doi:10.1007/s12028-014-9964-0

15. Saver JL, Starkman S, Eckstein M, et al. Prehospital use of magnesium sulfate as neuroprotection in acute stroke. $N$ Engl J Med. 2015;372(6):528-536. doi:10.1056/NEJMoa1408827

16. Huenges Wajer I, Dorhout Mees S, van den Bergh W, et al. Effect of magnesium on cognition after aneurysmal subarachnoid haemorrhage in a randomized trial. Eur J Neurol. 2018;25(12):1486-1489. doi:10.1111/ene.2018.25.issue-12

17. Sun L, Kosugi Y, Kawakami E, Piao YS, Hashimoto T, Oyanagi K. Magnesium concentration in the cerebrospinal fluid of mice and its response to changes in serum magnesium concentration. Magnes Res. 2009;22(4):266-272. doi:10.1684/mrh.2009.0186

18. Slutsky I, Abumaria N, Wu LJ, et al. Enhancement of learning and memory by elevating brain magnesium. Neuron. 2010;65(2):165177. doi:10.1016/j.neuron.2009.12.026

19. Li W, Yu J, Liu Y, et al. Elevation of brain magnesium prevents synaptic loss and reverses cognitive deficits in Alzheimer's disease mouse model. Mol Brain. 2014;7:65. doi:10.1186/s13041-014-0065-y

20. Sadir S, Tabassum S, Emad S, et al. Neurobehavioral and biochemical effects of magnesium chloride $(\mathrm{MgCl} 2)$, magnesium sulphate (MgSO 4) and magnesium-L-threonate (MgT) supplementation in rats: a dose dependent comparative study. Pak J Pharm Sci. 2019;32.

21. Gil-Martinez AL, Cuenca L, Sanchez C, Estrada C, FernandezVillalba E, Herrero MT. Effect of NAC treatment and physical activity on neuroinflammation in subchronic Parkinsonism; is physical activity essential? J Neuroinflammation. 2018;15(1):328. doi:10.1186/s12974-018-1357-4

22. Council NR. Guide for the Care and Use of Laboratory Animals. National Academies Press; 2010.

23. Li Y, Jiao Q, Du X, et al. Investigation of behavioral dysfunctions induced by monoamine depletions in a mouse model of parkinson's disease. Front Cell Neurosci. 2018;12:241. doi:10.3389/fncel.2018.00241

24. Miyanishi KC, Mohammed E, Watanabe M, Kubo M. Behavioral tests predicting striatal dopamine level in a rat hemi-Parkinson's disease model. Neurochem Int. 2019;122:8. doi:10.1016/j.neuint. 2018.11.005

25. Rozas G. The overall rod performance test in the MPTP-treatedmouse model of parkinsonism. J Neurosci Methods. 1998;83(2):10. doi:10.1016/S0165-0270(98)00078-8

26. Westermaier T, Stetter C, Kunze E, et al. Magnesium treatment for neuroprotection in ischemic diseases of the brain. Exp Transl Stroke Med. 2013;5(1):6. doi:10.1186/2040-7378-5-6

27. Lingam I, Meehan C, Avdic-Belltheus A, et al. Short-term effects of early initiation of magnesium infusion combined with cooling after hypoxia-ischemia in term piglets. Pediatr Res. 2019.

28. Gee JN. Hypermagnesemia does not increase brain intracellular magnesium in newborn swine. Pediatr Neurol. 2001;25(4):4. doi:10.1016/S0887-8994(01)00317-4

29. Pamphlett R. Magnesium supplementation does not delay disease onset or increase survival in a mouse model of familial ALS. $J$ Neurol Sci. 2003;216(1):3. doi:10.1016/S0022-510X(03)00216-8

30. Sen AP, Gulati A. Use of magnesium in traumatic brain injury. Neurotherapeutics. 2010;7(1):91-99. doi:10.1016/j.nurt.2009.10.014

31. McKee JA. Magnesium neuroprotection is limited in humans with acute brain injury. Neurocrit Care. 2005;2(3):9. doi:10.1385/ NCC:2:3:342
32. Euser AG, Cipolla MJ. Magnesium sulfate for the treatment of eclampsia: a brief review. Stroke. 2009;40(4):1169-1175. doi:10.1161/ STROKEAHA.108.527788

33. Wenwen X, Jing Y, Yingchao S, Qinglu W. The effect of magnesium deficiency on neurological disorders: a narrative review article. Iran J Public Health. 2019;48(3):379.

34. Li W, Yu J, Liu Y, et al. Elevation of brain magnesium prevents and reverses cognitive deficits and synaptic loss in Alzheimer's disease mouse model. Soc Neurosci. 2013;7:65.

35. Oyanagi K, Kawakami E, Kikuchi-Horie K, et al. Magnesium deficiency over generations in rats with special references to the pathogenesis of the parkinsonism-dementia complex and amyotrophic lateral sclerosis of Guam. Neuropathology. 2006;26(2):115-128. doi:10.1111/neu.2006.26.issue-2

36. Mazur A, Maier JA, Rock E, Gueux E, Nowacki W, Rayssiguier Y. Magnesium and the inflammatory response: potential physiopathological implications. Arch Biochem Biophys. 2007;458(1):48-56. doi:10.1016/j.abb.2006.03.031

37. Kharitonova M, Iezhitsa I, Zheltova A, Ozerov A, Spasov A, Skalny A. Comparative angioprotective effects of magnesium compounds. J Trace Elem Med Biol. 2015;29:227-234. doi:10.1016/j.jtemb.2014.06.026

38. Wolf FI, Trapani V, Simonacci M, Ferré S, Maier JA. Magnesium deficiency and endothelial dysfunction: is oxidative stress involved? Magnesium Res. 2008;21(1):58-64.

39. López A. Mitochondrial impairment and melatonin protection in parkinsonian mice do not depend of inducible or neuronal nitric oxide synthases. PLoS One. 2017;12(8):e0183090. doi:10.1371/journal.pone.0183090

40. Dzamko N, Geczy CL, Halliday GM. Inflammation is genetically implicated in Parkinson's disease. Neuroscience. 2015;302:89-102.

41. Kurosaki H, Yamaguchi K, Man-yoshi K, Muramatsu S-I, Hara S, Ichinose $\mathrm{H}$. Administration of tetrahydrobiopterin restored the decline of dopamine in the striatum induced by an acute action of MPTP. Neurochem Int. 2019;125:16-24. doi:10.1016/j.neuint.2019.02.005

42. Aras S, Tanriover G, Aslan M, Yargicoglu P, Agar A. The role of nitric oxide on visual-evoked potentials in MPTP-induced parkinsonism in mice. Neurochem Int. 2014;72:48-57. doi:10.1016/j.neuint.2014.04.014

43. Wu DC. Blockade of microglial activation is neuroprotective in the 1methyl-4-phenyl-1,2,3,6-tetrahydropyridine mouse model of parkinson disease. J Neurosci Methods. 2002;22(5):8.

44. Kim HG, Ju MS, Ha SK, et al. Acacetin protects dopaminergic cells against 1-methyl-4-phenyl-1,2,3,6-tetrahydropyridine-induced neuroinflammation in vitro and in vivo. Biol Pharm Bull. 2012;35 (8):1287-1294. doi:10.1248/bpb.b12-00127

45. Hammond SL. The Nurr1 Ligand,1,1-bis(3'-Indolyl)-1-(p-Chlorophenyl) methane, modulates glial reactivity and is neuroprotective in MPTPinduced parkinsonism. J Pharmacol Exp Ther. 2018;365(3):15. doi:10.1124/jpet.117.246389

46. Jiang L. Clozapine metabolites protect dopaminergic neurons through inhibition of microglial NADPH oxidase. J Neuroinflammation. 2016;13(1). doi:10.1186/s12974-016-0573-z

47. Barbagallo M, Belvedere M, Sprini D, Dominguez LJ. Magnesium and alzheimer's disease: implications for diet and nutrition. In: Martin CR, Preedy VR, editors. Diet and Nutrition in Dementia and Cognitive Decline. Elsevier; 2015:585-592.

48. Wang Q, Zhang H, Liu M, et al. [P38 MAPK signaling pathway regulates nuclear factor-kappaB and inducible nitric oxide synthase expressions in the substantia nigra in a mouse model of parkinson's disease]. Nan Fang Yi Ke Da Xue Xue Bao. 2014;34(8):1176-1180.

49. Ghosh A, Kanthasamy A, Joseph J, et al. Anti-inflammatory and neuroprotective effects of an orally active apocynin derivative in pre-clinical models of parkinson's disease. J Neuroinflammation. 2012;9:241. doi:10.1186/1742-2094-9-241

50. Tariq M, Khan HA, al Moutaery K, al Deeb SM. Effect of chronic administration of magnesium sulfate on 1-methyl-4-phenyl-1,2,3,6-tetrahydropyridine-induced neurotoxicity in mice. Pharmacol Toxicol. 1998;82(5):218-222. doi:10.1111/j.1600-0773.1998.tb01428.x 
51. Erro R, Pappatà S, Amboni M, et al. Anxiety is associated with striatal dopamine transporter availability in newly diagnosed untreated Parkinson's disease patients. Parkinsonism Relat Disord. 2012;18(9):1034-1038. doi:10.1016/j.parkreldis.2012.05.022

52. Wood SJ, Toth M. Molecular pathways of anxiety revealed by knockout mice. Mol Neurobiol. 2001;23(2-3):101-119. doi:10.1385/MN:23:2$3: 101$
53. Vuckovic MG, Wood RI, Holschneider DP, et al. Memory, mood, dopamine, and serotonin in the 1-methyl-4-phenyl-1,2,3,6-tetrahydropyridine-lesioned mouse model of basal ganglia injury. Neurobiol Dis. 2008;32(2):319-327. doi:10.1016/j.nbd.2008.07.015

\section{Publish your work in this journal}

Neuropsychiatric Disease and Treatment is an international, peerreviewed journal of clinical therapeutics and pharmacology focusing on concise rapid reporting of clinical or pre-clinical studies on a range of neuropsychiatric and neurological disorders. This journal is indexed on PubMed Central, the 'PsycINFO' database and CAS, and is the official journal of The International Neuropsychiatric Association (INA). The manuscript management system is completely online and includes a very quick and fair peer-review system, which is all easy to use. Visit http://www.dovepress.com/testimonials.php to read real quotes from published authors.

Submit your manuscript here: https://www.dovepress.com/neuropsychiatric-disease-and-treatment-journal 